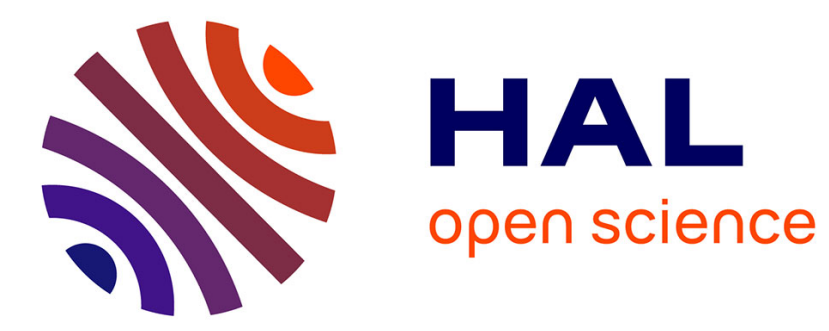

\title{
Robust control of a cable from a hyperbolic partial differential equation model
}

\author{
Lucie Baudouin, Aude Rondepierre, Simon Neild
}

\section{To cite this version:}

Lucie Baudouin, Aude Rondepierre, Simon Neild. Robust control of a cable from a hyperbolic partial differential equation model. IEEE Transactions on Control Systems Technology, 2019, 27 (3), pp.13431351. 10.1109/TCST.2018.2797938 . hal-01096851v2

\section{HAL Id: hal-01096851 \\ https://hal.science/hal-01096851v2}

Submitted on 22 Jan 2018

HAL is a multi-disciplinary open access archive for the deposit and dissemination of scientific research documents, whether they are published or not. The documents may come from teaching and research institutions in France or abroad, or from public or private research centers.
L'archive ouverte pluridisciplinaire $\mathbf{H A L}$, est destinée au dépôt et à la diffusion de documents scientifiques de niveau recherche, publiés ou non, émanant des établissements d'enseignement et de recherche français ou étrangers, des laboratoires publics ou privés. 


\title{
Robust control of a cable from a hyperbolic partial differential equation model
}

\author{
Lucie Baudouin, Aude Rondepierre and Simon Neild
}

\begin{abstract}
This paper presents a detailed study of the robust control of a cable's vibrations, with emphasis on considering a model of infinite dimension. Indeed, using a partial differential equation model of the vibrations of an inclined cable with sag, we are interested in studying the application of $\mathcal{H}_{\infty}$ robust feedback control to this infinite dimensional system. The approach relies on Riccati equations to stabilize the system under measurement feedback when it is subjected to external disturbances. Henceforth, our study focuses on the construction of a standard linear infinite dimensional state space description of the cable under consideration before writing its approximation of finite dimension and studying the $\mathcal{H}_{\infty}$ feedback control of vibrations with partial observation of the state in both cases. The closed loop system is numerically simulated to illustrate the effectiveness of the resulting control law.
\end{abstract}

Index Terms-Robust control, cable, partial differential equations, state-space model, measurement feedback.

\section{INTRODUCTION}

Inclined cables are common and critical components in a lot of civil engineering's structures and a large range of applications, from cable stayed bridges to telescopes and spacecraft [1]. Since cables are very flexible and lightly damped, one of the major issues related to such structures involving cables is the control of vibrations induced by any exterior perturbation. Their modeling is therefore very important in predicting and controlling the response to excitation. Many cable models exist, see [2] for instance. Of interest here is the modal formulation developed in [3] and partly validated experimentally in [4] and [5]. Vibration suppression in civil structures is also well documented, as in [6] or [7]. Passive dampers are the usual devices in civil structures but active control is potentially more effective and adaptive [8].

In this paper we study the design of robust control laws for a vibrating system composed of an inclined cable connected at its bottom end to an active control device in the framework of distributed parameter systems. More precisely, we work on a linearized model using partial differential equations (PDE) and choose a model-based feedback approach to disturbance rejection, namely the $\mathcal{H}_{\infty}$ measurement feedback control of the vibrating cable. Similar $\mathcal{H}_{\infty}$-approaches have been considered in [9] to suppress vibrations in flexible structures, but only in the finite dimensional setting. A preliminary version of the present study has been published in [10].

L. Baudouin is with LAAS-CNRS, 7 avenue du colonel Roche, F-31400 Toulouse, F-31400 Toulouse, France. Email: lucie.baudouin@laas.fr

A. Rondepierre is with IMT, University of Toulouse, INSA, Toulouse, France and LAAS-CNRS. Email: aude.rondepierre@math.univ-toulouse.fr

S. Neild is with Faculty of Engineering, University of Bristol, Queens Building, University Walk, Bristol BS8 1TR, UK. Email: simon.neild@bristol.ac.uk
Besides giving a theoretical robust control study based on a realistic model from civil engineering, the contribution of this paper is also to illustrate a theoretical result presented in [11] or [12] that gives the $\mathcal{H}_{\infty}$-robust control of infinite dimensional systems in terms of solvability of two coupled Riccati equations. Adopting this approach, we detail first the PDE modeling of the system so that it fits into the appropriate state-space framework. At this stage, from a non-linear system, we deduce a still meaningful linear system on which we actually work. Then, recalling the key aspects of the robust control theorem, we demonstrate that the required assumptions are met. Secondly, we perform numerical simulations. To this end, the infinite-dimensional robust control problem is approached by appropriate finite-dimensional ones. This earlylumping approach does not come along with a convergence result towards the theoretical infinite-dimensional result as in [13] since our observation operator will be unbounded. Finally, note that the robust stabilization of the linearized equation we will perform through this robust state space approach is not proved to imply the stabilization of the non-linear original system. This would be an interesting development for future research, considering that the robustness of our controller might handle the difficulties brought by the non-linearity.

We focus in Section III on the modeling of the inclined cable in the state-space framework. The first step is the construction of a mechanical model of the inclined cable, subject to gravitational effects (hence termed a cable rather than a string, corresponding to a situation without sag).

In a second step we describe how to control the cable system by the means of an active tendon, bringing active damping into the cable structure as in [14]. Lastly, the robust control problem is reformulated into an appropriate state-space framework. In Section III we first recall the $\mathcal{H}_{\infty}$ robust control theorem for infinite dimensional systems [11]. Then, this is applied to the cable control system once we prove the required assumptions in terms of stabilizability and detectability of the system. Section IV] is dedicated to numerical simulations.

Notations: The functional space of bounded linear operators from $E$ to $F$ (vector spaces) is denoted by $\mathcal{L}(E, F)$. The adjoint of an operator $A$ is denoted $A^{*}$. The space of square integrable functions is $L^{2}(0, \ell)$ and in $H_{0}^{1}(0, \ell)$, the functions need additionally to have a square integrable first weak derivative and a vanishing trace on the boundary. Then, $L^{\infty}(0,+\infty)$ is the functional space of essentially bounded functions. Finally, functions in $W^{2, \infty}(0,+\infty)$ are in $L^{\infty}(0,+\infty)$ as well as their two first weak derivatives. 


\section{INFINITE DIMENSIONAL MODEL}

As described in Figure 1, we consider a cable of length $\ell$, supported at end points $a$ and $b$, such that the direction of the chord line from $a$ to $b$ is defined as $x$, and the angle of inclination relative to the horizontal is denoted $\theta$.

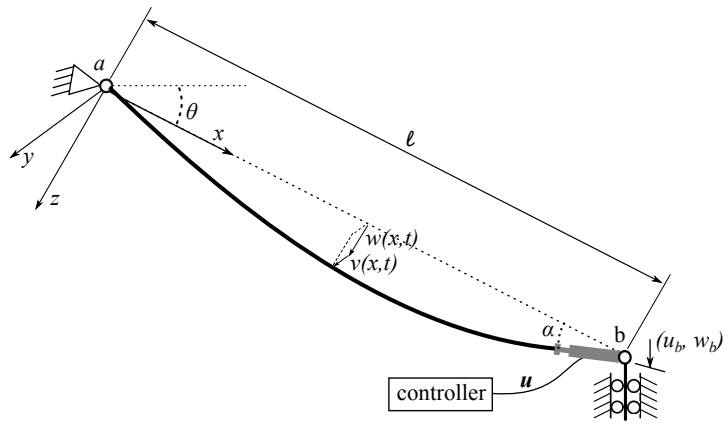

Fig. 1. Inclined Cable. See [15] Chapter 7].

Let $\rho$ be the density of the cable, $\mathcal{A}$ the cross-sectional area, $E$ Young's modulus and $g$ the gravity. We then define $\varrho=\rho g \cos \theta$ as the distributed weight perpendicular to the cable chord. The cable equilibrium sag position and the chord line both lie in the gravity plane, namely the $x z$-plane.

\section{A. Modeling of an inclined cable}

The modeling of an inclined cable presented hereafter is inspired from [15, sections 7.2 and 7.3], but the final equations of the motion are not exactly the same, since we put an emphasis on the perturbed dynamics rather than nonlinearity. Let us introduce some notations: $u(x, t)$ is the dynamic axial displacement of the cable (in $x$-direction) ; $v(x, t)$ is the dynamic out-of-plane transverse displacement (in $y$-direction) ; $w(x, t)$ is the dynamic in-plane transverse displacement (in $z$-direction) ; $T_{s}$ is the static tension of the cable (assumed constant w.r.t. $(x, t)) ; w_{s}(x)=\varrho \mathcal{A}\left(\ell x-x^{2}\right) / 2 T_{s}$ is the static in-plane displaced shape of the cable. Note that the sag is assumed small in comparison to the length of the cable, but still affects the static deflexion of the cable so that $w_{s}$ could be calculated precisely [15]; $T(x, t)$ is the dynamic tension of the cable. As long as the cable remains within its elastic range, one has:

$$
T=\mathcal{A} E\left[\partial_{x} u+\frac{1}{2}\left(\partial_{x} v\right)^{2}+\frac{1}{2}\left(\partial_{x} w\right)^{2}+\frac{d w_{s}}{d x} \partial_{x} w\right]
$$

Next the main steps of the description of our model will be: the boundary conditions, the linearization of the dynamic tension, the equations of motion of the cable and the focus on the in-plane dynamic and its decomposition in order to obtain finally a PDE that will be the object of our theoretical study.

The inclined cable is excited vertically at its lower end. This yields the following boundary conditions corresponding to the support motion: for all $t>0$,

$$
\left\{\begin{array}{lll}
u(0, t)=0, & v(0, t)=0, & w(0, t)=0, \\
u(\ell, t)=u_{b}(t), & v(\ell, t)=0, & w(\ell, t)=w_{b}(t) .
\end{array}\right.
$$

To satisfy these time-varying conditions, the cable response is decomposed into a quasi-static component (denoted by the subscript $q$ ) which corresponds to the displacements of the cable moving as an elastic tendon due to support movement, and satisfies the boundary conditions (1), and a modal component (denoted by the subscript $m$ ) capturing the dynamic response of the cable with fixed ends (boundary conditions equal to 0 ).

Let us now focus on the equations of motion of the cable. In [15], these equations are linearized enabling the authors to completely decouple the quasi-static and modal terms under the assumption that both motions are small compared with the static sag. Here we choose a slightly different approach: the non-linearities of the cable dynamics are also ignored in order to fit to the linear infinite dimensional state space framework. But we write and solve the quasi-static equations of motion and then reinject these solutions in the complete equations of motion to obtain the modal PDE.

Let us first linearize the dynamic tension: for all $(x, t)$ in $(0, \ell) \times(0, \infty)$

$$
T(x, t)=\mathcal{A} E\left[\partial_{x} u(x, t)+\frac{d w_{s}}{d x}(x) \partial_{x} w(x, t)\right] .
$$

We further assume that there is no significant dynamic response along the $x$-axis (meaning in particular $u_{m}=0$ ) as the axial vibrations are usually excluded from models since the frequency of oscillations is much faster and of smaller amplitude than that in the other directions. Assuming finally that the linearized dynamic tension is small compared to the static tension $\left(T \ll T_{s}\right.$ ), the equations of motion for the inclined cable are given, for all $(x, t)$ in $(0, \ell) \times(0, \infty)$, by:

$$
\begin{aligned}
& \rho \mathcal{A} \partial_{t t} v(x, t)=T_{s} \partial_{x x} v(x, t), \\
& \rho \mathcal{A} \partial_{t t} w(x, t)=T_{s} \partial_{x x} w(x, t)+T(x, t) \frac{d^{2} w_{s}}{d x^{2}} .
\end{aligned}
$$

Observe that when linearizing the dynamic tension of the cable, we lost the sole coupling between $v$ and $w$. The out-of-plane motion $v$ satisfies a conservative wave equation that could only be influenced by coupling nonlinearities not considered here. Since the control and the perturbations will only act in the gravity plane $(x z)$, the out-of-plane motion $v$ is not considered as a part of our control system anymore, and will not appear in the construction of our state space model. As a consequence, the remaining equation, of unknown $w$, looks like the one of a horizontal cable (for which $\theta=0$ ).

We now focus on the in-plane motion for the dynamic analysis of the inclined cable following equation (3) along with the boundary conditions (1) and some appropriate initial data. As previously mentioned, we first solve the quasistatic equations of the cable, with time dependent boundary conditions i.e. precisely: for all $(x, t)$ in $(0, \ell) \times(0, \infty)$ :

$$
\left\{\begin{array}{l}
T_{q}=\mathcal{A} E\left[\partial_{x} u_{q}+\frac{d w_{s}}{d x}(x) \partial_{x} w_{q}\right] \\
T_{s} \partial_{x x} w_{q}+T_{q} \frac{d^{2} w_{s}}{d x^{2}}=0 \\
u_{q}(0)=w_{q}(0) \stackrel{2}{=} 0, u_{q}(\ell)=u_{b}, w_{q}(\ell)=w_{b},
\end{array}\right.
$$

As detailed in [15], the quasi-static equations (4) have the following solutions:

$$
w_{q}(x, t)=w_{b}(t) \frac{x}{\ell}-\frac{\varrho E_{q} \ell \mathcal{A}^{2}}{2 T_{s}^{2}} u_{b}(t)\left[\frac{x}{\ell}-\left(\frac{x}{\ell}\right)^{2}\right]
$$




$$
\begin{aligned}
u_{q}(x, t)= & \frac{E_{q}}{E} u_{b}(t) \frac{x}{\ell}-\frac{\varrho \mathcal{A} \ell}{2 T_{s}} w_{b}(t)\left[\frac{x}{\ell}-\left(\frac{x}{\ell}\right)^{2}\right] \\
& +\frac{\lambda^{2} E_{q}}{4 E} u_{b}(t)\left[\frac{x}{\ell}-2\left(\frac{x}{\ell}\right)^{2}+\frac{4}{3}\left(\frac{x}{\ell}\right)^{3}\right] \\
T_{q}(t)= & \frac{\mathcal{A} E_{q}}{\ell} u_{b}(t)
\end{aligned}
$$

where $E_{q}=E /\left(1+\lambda^{2} / 12\right)$ is the equivalent modulus of the cable and $\lambda^{2}=E \varrho^{2} \ell^{2} \mathcal{A}^{3} / T_{s}^{3}$ the Irvine's parameter.

Then let $T_{m}=T-T_{q}, u_{m}=u-u_{q}$ and $w_{m}=w-w_{q}$. Since $u_{m}=0$, the modal dynamic tension satisfies

$$
T_{m}=\mathcal{A} E \frac{d w_{s}}{d x} \partial_{x} w_{m}=\frac{\varrho \mathcal{A}^{2} E}{2 T_{s}}(\ell-2 x) \partial_{x} w_{m}
$$

and from (3) and (4), the in-plane modal displacement $w_{m}$ is solution of the following PDE on $(0, \ell) \times(0, \infty)$ :

$$
\rho \mathcal{A} \partial_{t t}\left(w_{q}+w_{m}\right)=T_{s} \partial_{x x} w_{m}+T_{m} \frac{d^{2} w_{s}}{d x^{2}},
$$

subject to homogeneous Dirichlet boundary conditions $w_{m}(0, t)=0, w_{m}(\ell, t)=0$ for all $t \in(0, \infty)$ and initial conditions equal to zero.

Since $\partial_{t t} w_{q}$ is easily calculated from (5) and: $d^{2} w_{s} / d x^{2}=$ $-\varrho \mathcal{A} / T_{s}$, we get the self-contained equation on $(0, \ell) \times(0, \infty)$ :

$$
\begin{gathered}
\partial_{t t} w_{m}=\frac{T_{s}}{\rho \mathcal{A}} \partial_{x x} w_{m}-\frac{\varrho^{2} \mathcal{A}^{2} E}{2 \rho T_{s}^{2}}(\ell-2 x) \partial_{x} w_{m} \\
-\frac{x}{\ell} w_{b}^{\prime \prime}+\frac{\varrho E_{q} \ell \mathcal{A}^{2}}{2 T_{s}^{2}}\left[\frac{x}{\ell}-\left(\frac{x}{\ell}\right)^{2}\right] u_{b}^{\prime \prime} .
\end{gathered}
$$

Remark 1: This formulation of the in-plane motion dynamic of the cable ensures that the disturbances $u_{b}, w_{b}$ no longer enter the model as boundary conditions as in (1). Instead, they appear in (6) in a way that will be represented by a bounded control operator [16]. As a related question, the stabilization of a simplified hyperbolic model is studied in [17] by a backstepping approach.

\section{B. Modeling of the measurement and control terms}

The inclined cable device depicted in Figure 1, is perturbed by in-plane oscillations $\left(u_{b}, w_{b}\right)$ and connected at its bottom end with an active tendon. Using a support motion at the cable's anchorage is a natural choice of active control since the installation of the proper device can be done with small modifications of the lower end of the cable, [8]. Moreover, we aim to obtain good results when considering robust control with partial observation using an active tendon since the collocation of actuator and sensor has proved great effectiveness in active damping of cables, [14] and [6].

An active tendon can be described as a displacement actuator collocated with a force sensor (see e.g. [18]). Therefore, on the one hand, the force sensor allows us to define the dynamic tension at the location of the tendon $T(\ell, t)$ as the measurement we have to build our feedback. On the other hand, even if the action of a tendon of amplitude $\mathbf{u}$ is principally meant to be an axial movement [7], a careful consideration of the projection of the tendon's displacement on the $x$ and $z$-axis shows that its action can be written in terms of the angle $\alpha$ it makes with the chord line (see Fig. 11). It gives a control of coordinates $(\mathbf{u} \cos \alpha, \mathbf{u} \sin \alpha)$, approximated in two different contributions in equation (6) of form $\alpha \mathbf{u}^{\prime \prime}$ added to $u_{b}^{\prime \prime}$ and $\left(1-\frac{\alpha^{2}}{2}\right) \mathbf{u}^{\prime \prime}$ added to $w_{b}^{\prime \prime}$.

Let us now translate this information into the equations. We consider the following state equation on $(0, \ell) \times(0, \infty)$, controlled by the scalar input $\mathbf{u}^{\prime \prime}$ (noting $\sigma=\varrho E_{q} \ell \mathcal{A}^{2} / 2 T_{s}^{2}$ ):

$$
\begin{array}{r}
\partial_{t t} w_{m}=\frac{T_{s}}{\rho \mathcal{A}} \partial_{x x} w_{m}-\frac{\varrho^{2} \mathcal{A}^{2} E}{2 \rho T_{s}^{2}}(\ell-2 x) \partial_{x} w_{m}-\xi \partial_{t} w_{m} \\
+\sigma\left[\frac{x}{\ell}-\frac{x^{2}}{\ell^{2}}\right]\left(u_{b}^{\prime \prime}+\left(1-\frac{\alpha^{2}}{2}\right) \mathbf{u}^{\prime \prime}\right)-\frac{x}{\ell}\left(w_{b}^{\prime \prime}+\alpha \mathbf{u}^{\prime \prime}\right)
\end{array}
$$

with the information of the localized measurement output

$$
T(x=\ell)=T_{q}+T_{m}(\ell)=\frac{\mathcal{A} E_{q}}{\ell} u_{b}-\frac{\varrho \mathcal{A}^{2} E \ell}{2 T_{s}} \partial_{x} w_{m}(\ell) .
$$

A realistic viscous damping term $\xi \partial_{t} w_{m}$ has been added to our hyperbolic PDE, $\xi$ being a positive diagonal bounded operator that will take the shape of a modal damping when translated in the finite dimensional system build in Section IV

Remark 2: Using the denominations from [8], [7], the axial part (along $u_{b}$ ) of the control is actually an inertial control proportional to $\mathbf{u}^{\prime \prime}$, and if we had this sole contribution, we would only have access to the symmetric modes of vibration. A parametric control takes the shape $\mathbf{u} w_{m}$ and gives access to the control of all the vibration modes. But our linearized framework has lost track of this bilinear control. Luckily, the alignment defect of the active tendon with the cable's chord gives a contribution to the in-plane lower support displacement as a small proportion of $\mathbf{u}^{\prime \prime}$ added to the perturbation $w_{b}$.

\section{State space model of the robust control system}

Let $X=\left(w_{m}, \partial_{t} w_{m}\right)$ be the state and $W=\left(W_{\text {mod }}, u_{b}, w_{b}^{\prime \prime}\right)$ the exogenous disturbance where $W_{\text {mod }}$ gathers uncertainty on the model (e.g. the neglected nonlinearities). Let $u_{b}^{\prime \prime}=-\omega_{u}^{2} u_{b}$ and the control input $U=\mathbf{u}^{\prime \prime}$ be the acceleration of the displacement actuator. The measurement output $Y=T(\ell, \cdot)$ is given by the force sensor and the "to be controlled" output $Z$ will be chosen later according to the robust control objectives.

The linear infinite-dimensional state-space model takes the usual shape [19]: for all $t>0$,

$$
\left\{\begin{array}{l}
X^{\prime}(t)=A X(t)+B_{1} W(t)+B_{2} U(t), \\
Z(t)=C_{1} X(t)+D_{12} U(t), \\
Y(t)=C_{2} X(t)+D_{21} W(t),
\end{array}\right.
$$

with $X(0)=0$. Mainly based on equations (7)-(8), the operator matrices involved in (9) are given by:

$$
\begin{aligned}
& A=\left(\begin{array}{cc}
0 & I \\
\frac{T_{s}}{\rho \mathcal{A}} \partial_{x x}-\frac{\varrho^{2} \mathcal{A}^{2} E}{2 \rho T_{s}^{2}}(\ell-2 x) \partial_{x} & -\xi
\end{array}\right), \\
& B_{1}=\left(\begin{array}{ccc}
0 & 0 & 0 \\
d^{1} & -\omega_{u}^{2} \frac{\varrho E_{q} \ell \mathcal{A}^{2}}{2 T_{s}^{2}}\left[\frac{x}{\ell}-\left(\frac{x}{\ell}\right)^{2}\right] & -\frac{x}{\ell}
\end{array}\right),
\end{aligned}
$$




$$
\begin{gathered}
B_{2}=\left(\left(1-\frac{\alpha^{2}}{2}\right) \frac{\varrho E_{q} \ell \mathcal{A}^{2}}{2 T_{s}^{2}}\left[\frac{x}{\ell}-\left(\frac{x}{\ell}\right)^{2}\right]-\alpha \frac{x}{\ell}\right), \\
C_{2}=\left(\begin{array}{ll}
-\left.\frac{\varrho \mathcal{A}^{2} E \ell}{2 T_{s}} \partial_{x} \cdot\right|_{x=\ell} & 0
\end{array}\right), D_{21}=\left(\begin{array}{lll}
d^{2} & \frac{\mathcal{A} E_{q}}{\ell} & 0
\end{array}\right),
\end{gathered}
$$

where $d^{1}$ and $d^{2}$ are tuning parameters and $\xi$ is the modal damping operator. Then, depending on the control objectives of performance, we can choose for instance $Z=\left(w_{m}, \mathbf{u}^{\prime \prime}\right)$, i.e. $C_{1}=\left(\begin{array}{ll}I & 0 \\ 0 & 0\end{array}\right), D_{12}=\left(\begin{array}{l}0 \\ I\end{array}\right)$ to describe the objective of reducing the in-plane movement of the cable, while limiting the amplitude of the control. Different objectives will be studied in numerical simulations later on.

Let us now define the appropriate functional Hilbert spaces associated with the infinite-dimensional model. The state space is given by $\mathcal{X}=H_{0}^{1}(0, \ell) \times L^{2}(0, \ell)$, the input or output spaces are: $\mathcal{U}=\mathbb{R}, \mathcal{W}=\mathbb{R}^{3}, \mathcal{Y}=\mathbb{R}, \mathcal{Z}=H_{0}^{1}(0, \ell) \times \mathbb{R}$. The Hilbert space $\mathcal{X}$ is equipped with the scalar product:

$$
\left\langle\left(\begin{array}{l}
f_{1} \\
g_{1}
\end{array}\right),\left(\begin{array}{l}
f_{2} \\
g_{2}
\end{array}\right)\right\rangle_{\mathcal{X}}=\left\langle\partial_{x} f_{1}, \partial_{x} f_{2}\right\rangle_{L^{2}}+\left\langle g_{1}, g_{2}\right\rangle_{L^{2}}
$$

We prove hereafter that the operator $A$ of domain $\mathcal{D}(A)=$ $\left(H^{2} \cap H_{0}^{1}\right)(0, \ell) \times H_{0}^{1}(0, \ell)$ is the infinitesimal generator of a $C_{0}$-semigroup $T(t)=e^{A t}$ on the space $\mathcal{X}$ and operators $B_{1} \in$ $\mathcal{L}(\mathcal{W}, \mathcal{X}), B_{2} \in \mathcal{L}(\mathcal{U}, \mathcal{X}), C_{1} \in \mathcal{L}(\mathcal{X}, \mathcal{Z}), D_{12} \in \mathcal{L}(\mathcal{U}, \mathcal{Z})$ and $D_{21} \in \mathcal{L}(\mathcal{W}, \mathcal{Y})$ are bounded.

We use the classical theory of semi-groups to study the operator $A$. Since $-\partial_{x x}$ is a self-adjoint, non-negative and coercive operator, we can write $A=A_{0}+P$ where

$A_{0}=\left(\begin{array}{ll}0 & I \\ \frac{T_{s}}{\rho \mathcal{A}} \partial_{x x} & 0\end{array}\right)$ and $P=\left(\begin{array}{cc}0 & 0 \\ -\frac{\varrho^{2} \mathcal{A}^{2} E}{2 \rho T_{s}^{2}}(\ell-2 x) \partial_{x} & -\xi\end{array}\right)$

are such that $A_{0}$ is the infinitesimal generator of a $C_{0^{-}}$ semigroup (see [16, chapter 2.2] or [20, chapter 2.7]) and $P$ is a linear bounded perturbation of it (see the last remark in [20, chapter 7.3]). Thus, $A$ is the infinitesimal generator of a $C_{0^{-}}$ semigroup and the PDE interpretation of the above semi-group goes as follows:

Under any initial data $w_{m}(t=0)=w_{0} \in H_{0}^{1}(0, \ell)$ and $\partial_{t} w_{m}(t=0)=w_{1} \in L^{2}(0, \ell)$, assuming that $u_{b}, w_{b}$ and $\mathbf{u}$ belong to $W^{2, \infty}(0,+\infty)$ and that $\xi \in \mathcal{L}\left(L^{2}(0, \ell) ;\right] 0,+\infty[)$, there exists a unique solution to the initial and homogeneous boundary value problem given by equation (7), such that

$$
w_{m} \in C\left(\mathbb{R}_{+} ; H_{0}^{1}(0, \ell)\right) \cap C^{1}\left(\mathbb{R}_{+} ; L^{2}(0, \ell)\right) .
$$

Observe that as long as we rely only on a boundary observation (at $x=\ell$ ) of the cable's tension, the measurement output operator $C_{2}$ does not belong to $\mathcal{L}(\mathcal{X}, \mathcal{Y})$. Instead, since $H^{1}(0, \ell) \subset C([0, \ell])$, we have: $C_{2} \in \mathcal{L}(\mathcal{D}(A), \mathcal{Y})$ i.e. there exists $M>0$ such that for all $(f, g) \in \mathcal{D}(A)$,

$$
\begin{aligned}
\left\|C_{2}\left(\begin{array}{l}
f \\
g
\end{array}\right)\right\|_{\mathcal{Y}} & =\frac{\varrho \mathcal{A}^{2} E \ell}{2 T_{s}}\left|\partial_{x} f(\ell)\right| \leq M\left\|\partial_{x} f\right\|_{H^{1}(0, \ell)} \\
& \leq M\|f\|_{H^{2}(0, \ell)} \leq M\|(f, g)\|_{\mathcal{D}(A)}
\end{aligned}
$$

\section{ROBUST CONTROL ISSUES}

We first recall here a theorem proved in [21] and revisited in [11, [12] or [22] that we will apply then to the PDE model derived in Section III This result gives an equivalence between the $\mathcal{H}_{\infty}$-robust control with measurement-feedback of a PDE system and the solvability of two Riccati equations. We specifically refer to [11] and [22] for the case of unbounded observation operator as it is our situation here.

\section{A. $\mathcal{H}_{\infty}$-control with measurement feedback}

Assume that $A$ is the infinitesimal generator of a $C_{0^{-}}$ semigroup on the space $\mathcal{X}$ and $B_{1}, B_{2}, C_{1}, C_{2}, D_{12}$ and $D_{21}$ are bounded operators (or even unbounded, as $C_{2}$ for instance, see [22] or [23]) in the appropriate functional spaces. Without loss of generality, we make the classical normalization assumptions $D_{12}^{*}\left[\begin{array}{ll}C_{1} & D_{12}\end{array}\right]=\left[\begin{array}{ll}\mathbf{0} & I\end{array}\right]$ and $D_{21}\left[\begin{array}{ll}D_{21}^{*} & B_{1}^{*}\end{array}\right]=$ $\left[\begin{array}{ll}I & \mathbf{0}\end{array}\right]$, in order to simplify the formulation of the problem.

The state-space description (9) of the system allows the control of the state from the knowledge of the partial observation $Y=C_{2} X+D_{21} W$ and under the cost function $J_{0}(U, W)=\int_{0}^{\infty}\left(\left\|C_{1} X(t)\right\|_{\mathcal{Z}}^{2}+\|U(t)\|_{\mathcal{U}}^{2}\right) d t$. The objective is to construct a dynamic measurement-feedback controller $\mathcal{K}=\left(A_{\mathcal{K}}, B_{\mathcal{K}}, C_{\mathcal{K}}, D_{\mathcal{K}}\right)$ of shape, for all $t>0$,

$$
\left\{\begin{array}{l}
\Phi^{\prime}(t)=\left(A+A_{\mathcal{K}}\right) \Phi(t)+B_{\mathcal{K}} Y(t) \\
U(t)=C_{\mathcal{K}} \Phi(t)+D_{\mathcal{K}} Y(t)
\end{array}\right.
$$

with $\Phi(0)=0$, that exponentially stabilize the coupled system:

$$
\left\{\begin{array}{l}
X^{\prime}=\left(A+B_{2} D_{\mathcal{K}} C_{2}\right) X+B_{2} C_{\mathcal{K}} \Phi+\left(B_{1}+B_{2} D_{\mathcal{K}} D_{21}\right) W \\
\Phi^{\prime}=B_{\mathcal{K}} C_{2} X+\left(A+A_{\mathcal{K}}\right) \Phi+B_{\mathcal{K}} D_{21} W
\end{array}\right.
$$

in closed loop and ensures that the influence of the disturbances on the "to be controlled output" $Z$ is smaller than some specific bound $\gamma$. Let us introduce the operator:

$$
\Lambda=\left(\begin{array}{cc}
A+B_{2} D_{\mathcal{K}} C_{2} & B_{2} C_{\mathcal{K}} \\
B_{\mathcal{K}} C_{2} & A+A_{\mathcal{K}}
\end{array}\right)
$$

The result we will apply to the feedback control of a cable is the following:

Theorem 1: Let $\gamma>0$ and assume that the pair $\left(A, B_{1}\right)$ is stabilizable, the pair $\left(A, C_{1}\right)$ is detectable and assume that $C_{2}$ is an admissible operator. These assertions are equivalent: (i) The $\gamma^{2}$-robustness property with partial observation holds for the system (9): there exists an exponentially stabilizing dynamic output-feedback controller $\mathcal{K}$ of the form $(10)$ such that $\Lambda$ is exponentially stable and $\rho(\mathcal{K})<\gamma^{2}$.

(ii) There exist two nonnegative definite symmetric operators $P, \Sigma \in \mathcal{L}(\mathcal{X})$ solutions of the Riccati and compatibility equations:

- $A-\left(B_{2} B_{2}^{*}-\gamma^{-2} B_{1} B_{1}^{*}\right) P$ generates an exponentially stable semigroup and $\forall X \in \mathcal{D}(A), P X \in \mathcal{D}\left(A^{*}\right)$ and $\left(P A+A^{*} P-P\left(B_{2} B_{2}^{*}-\gamma^{-2} B_{1} B_{1}^{*}\right) P+C_{1}^{*} C_{1}\right) X=0$;

- $A^{*}-\left(C_{2}^{*} C_{2}-\gamma^{-2} C_{1}^{*} C_{1}\right) \Sigma$ generates an exponentially stable semigroup and $\forall X \in \mathcal{D}\left(A^{*}\right), \Sigma X \in \mathcal{D}(A)$ and $\left(\Sigma A^{*}+A \Sigma-\Sigma\left(C_{2}^{*} C_{2}-\gamma^{-2} C_{1}^{*} C_{1}\right) \Sigma+B_{1} B_{1}^{*}\right) X=0$; - $I-\gamma^{-2} P \Sigma$ is invertible, $\Pi=\Sigma\left(I-\gamma^{-2} P \Sigma\right)^{-1} \geq 0$. 
Moreover, if these three conditions hold, then the feedback controller $\mathcal{K}$ specified by

$$
\begin{aligned}
& A_{\mathcal{K}}=-\left(B_{2} B_{2}^{*}-\gamma^{-2} B_{1} B_{1}^{*}\right) P-\Pi C_{2}^{*} C_{2} \\
& B_{\mathcal{K}}=\Pi C_{2}^{*}, C_{\mathcal{K}}=-B_{2}^{*} P, D_{\mathcal{K}}=0,
\end{aligned}
$$

gives an exponentially stable operator $\Lambda$ and guarantees that $\rho(\mathcal{K})<\gamma^{2}$. Finally, if the solutions to the Riccati equations exist, then they are unique.

The definitions of stabilizability or exponentially stability can be found in [16]. The feedback controller $\mathcal{K}$, known as the central controller, is actually sub-optimal. We rely on the proof (among others, e.g. [13], [22]) that can be read in [11], since we deal with an unbounded yet admissible observation operator $C_{2}$ (to have a Pritchard-Salamon system). Reference [23] is specific to operator $B_{2}$ unbounded, and [12] or [21] to the bounded operator case.

\section{B. Admissibility, controllability and observability assumptions}

This subsection is devoted to the verification of the stabilizability, detectability and admissibility assumptions needed to apply Theorem 1 in the context of the inclined cable. Since exact controllability implies exponential stabilizability, as well as exact observability implying exponential detectability [16], we actually focus instead on these specific properties. In fact, using [24], we precisely obtain that the wave equation $X^{\prime}=A X+B_{1} W$ is exactly controllable through $W=$ $\left(W_{\text {mod }}, u_{b}, w_{b}^{\prime \prime}\right)$, which implies the exponential stabilizability of the pair $\left(A, B_{1}\right)$. The specificity of the controllability result we need relies on the force distribution functions (e.g. $x \mapsto-x / \ell$ ) through which the controls (e.g. $w_{b}^{\prime \prime}$ ) are acting on the cable. On the other hand, the exponential detectability of the pair $\left(A, C_{1}\right)$ will stem from the exact observability property easily proved through the method described in [20].

1) Observability of the pair $\left(A, C_{1}\right)$ : It can be deduced (see [20]) from the observability of the simplified (undamped, unperturbed and normalized) pair

$$
\left(A_{0}=\left(\begin{array}{cc}
0 & I \\
\partial_{x x} & 0
\end{array}\right), C_{0}=\left(\begin{array}{ll}
I & 0
\end{array}\right)\right) .
$$

Indeed lower order terms in the wave equation, as the ones gathered in the perturbation operator, are known, in general, not to affect the observability/controllability results (see e.g. [25]).

On one hand, defining $\mathcal{D}(A)=H^{2}(0, \ell) \cap H_{0}^{1}(0, \ell) \times$ $H_{0}^{1}(0, \ell)$, we have $A_{0}:(f, g) \in \mathcal{D}(A) \mapsto\left(g, \partial_{x x} f\right) \in \mathcal{X}$, whose eigenvalues are $\lambda_{n}=i n \pi / \ell$ and eigenvectors take the shape, for all $n \in \mathbb{Z}^{*}$ :

$$
\phi_{n}=\frac{1}{\sqrt{2}}\left(\begin{array}{c}
\frac{\ell}{i n \pi} \varphi_{n} \\
\varphi_{n}
\end{array}\right), \varphi_{n}=\sqrt{\frac{2}{\ell}} \sin \left(\frac{n \pi x}{\ell}\right) .
$$

The operator $A_{0}$ generates a unitary group $\mathcal{T}_{0}$ on $\mathcal{X}$ (e.g. semigroup theory or separation principle) given by, for $(f, g) \in \mathcal{X}$ :

$$
\begin{aligned}
& \mathcal{T}_{0}(t)\left(\begin{array}{c}
f \\
g
\end{array}\right)=\sum_{n \in \mathbb{Z}^{*}} e^{\lambda_{n} t}\left\langle\left(\begin{array}{l}
f \\
g
\end{array}\right), \phi_{n}\right\rangle_{\mathcal{X}} \phi_{n} \\
& =\frac{1}{2} \sum_{n \in \mathbb{Z}^{*}} e^{\frac{i n \pi}{\ell} t}\left(i\left\langle\partial_{x} f, \psi_{n}\right\rangle_{L^{2}}+\left\langle g, \varphi_{n}\right\rangle_{L^{2}}\right)\left(\begin{array}{c}
\frac{\ell}{i n \pi} \varphi_{n} \\
\varphi_{n}
\end{array}\right) .
\end{aligned}
$$

where $\psi_{n}=\sqrt{\frac{2}{\ell}} \cos \left(\frac{n \pi x}{\ell}\right)$ for all $n \in \mathbb{Z}$. On the other hand, $C_{0} \in \mathcal{L}\left(\mathcal{X}, H_{0}^{1}(0, \ell)\right)$ is defined by $C_{0}:(f, g) \in \mathcal{X} \mapsto f \in$ $H_{0}^{1}(0, \ell)$, and it is easy to prove that the pair $\left(A_{0}, C_{0}\right)$ is exactly observable in time $T>2 \ell$. It requires e.g. (see [20]) to prove there exists $k>0$ such that for all $(f, g) \in \mathcal{D}(A)$,

$$
\mathcal{C}:=\int_{0}^{2 \ell}\left\|C_{0} \mathcal{T}_{0}(t)\left(\begin{array}{l}
f \\
g
\end{array}\right)\right\|_{H_{0}^{1}(0, \ell)}^{2} d t \geq k\left\|\left(\begin{array}{l}
f \\
g
\end{array}\right)\right\|_{\mathcal{X}}^{2} .
$$

Using $\left\|\frac{\ell}{i n \pi} \varphi_{n}\right\|_{H_{0}^{1}(0, \ell)}=1$ and the orthonormality of the family $\left\{t \mapsto \frac{\exp \left(\frac{i n \pi}{\ell} t\right)}{\sqrt{2 \ell}}, n \in \mathbb{Z}^{*}\right\}$ in $L^{2}(0,2 \ell)$, we get

$$
\begin{aligned}
4 \mathcal{C} & =\int_{0}^{2 \ell}\left\|\sum_{n \in \mathbb{Z}^{*}} e^{\frac{i n \pi}{\ell} t}\left(i\left\langle\partial_{x} f, \psi_{n}\right\rangle_{L^{2}}+\left\langle g, \varphi_{n}\right\rangle_{L^{2}}\right) \frac{\ell \varphi_{n}}{i n \pi}\right\|_{H_{0}^{1}}^{2} d t \\
& =2 \ell \sum_{n \in \mathbb{Z}^{*}}\left|i\left\langle\partial_{x} f, \psi_{n}\right\rangle_{L^{2}}+\left\langle g, \varphi_{n}\right\rangle_{L^{2}}\right|^{2} .
\end{aligned}
$$

From $\varphi_{-n}=-\varphi_{n}, \psi_{-n}=\psi_{n}$ and the parallelogram identity $\left(|a+b|^{2}+|a-b|^{2}=2|a|^{2}+2|b|^{2}\right)$, it follows

$$
\mathcal{C}=\ell \sum_{n \in \mathbb{N}}\left(\left|\left\langle\partial_{x} f, \psi_{n}\right\rangle_{L^{2}}\right|^{2}+\left|\left\langle g, \varphi_{n}\right\rangle_{L^{2}}\right|^{2}\right)=\ell\left\|\left(\begin{array}{l}
f \\
g
\end{array}\right)\right\|_{\mathcal{X}}^{2}
$$

since the families $\left\{\psi_{n}, n \in \mathbb{N}\right\}$ and $\left\{\varphi_{n}, n \in \mathbb{N}^{*}\right\}$ are hilbertian (orthonormal) basis of $L^{2}(0, \ell)$, implying (13).

2) Admissibility of the observation operator $C_{2}$ : according to [20], $C_{2} \in \mathcal{L}(\mathcal{D}(A), \mathcal{Y})$ is admissible for $\mathcal{T}_{0}$ if and only if for some $\tau>0$, there exists a constant $k_{\tau} \geq 0$ such that for all $(f, g) \in \mathcal{D}(A), \int_{0}^{\tau}\left\|C_{2} \mathcal{T}_{0}(t)(f, g)\right\|_{\mathcal{Y}}^{2} d t \leq k_{\tau}\|(f, g)\|_{\mathcal{X}}^{2}$. Following the exact same steps as in the previous paragraph, we can calculate that $\int_{0}^{2 \ell}\left|C_{2} \mathcal{T}_{0}(t)\left(\begin{array}{l}f \\ g\end{array}\right)\right|^{2} d t=2\left\|\left(\begin{array}{l}f \\ g\end{array}\right)\right\|_{\mathcal{X}}^{2}$, thus obtaining the admissibility of $C_{2}$ and the detectability of the pair $\left(A, C_{2}\right)$.

3) Stabilizability of the pair $\left(A, B_{1}\right)$ : applying the same method to prove the stabilizability of the pair $\left(A, B_{1}\right)$, the best we obtain is strong stabilizability, instead of exponential stabilizability. As mentioned before, the exact observability of the dual pair $\left(A^{*}, B_{1}^{*}\right)$ can be deduced from the exact observability of the simplified (undamped and unperturbed) pair $\left(A_{0}, B_{0}^{*}\right), A_{0}$ being skew-adjoint, and $B_{0}$ satisfying

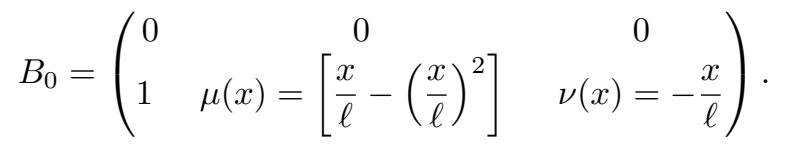

One can easily calculate for all $W \in \mathbb{R}^{3}$ and all $(f, g) \in \mathcal{X}$,

$$
\begin{aligned}
& \left\langle B_{0} W,\left(\begin{array}{l}
f \\
g
\end{array}\right)\right\rangle_{\mathcal{X}}=\left\langle W_{\text {mod }}+\mu u_{b}+\nu w_{b}^{\prime \prime}, g\right\rangle_{L^{2}} \\
& =\left\langle W, B_{0}^{*}\left(\begin{array}{l}
f \\
g
\end{array}\right)\right\rangle_{\mathbb{R}^{3}} \text { where } B_{0}^{*}=\left(\begin{array}{cc}
0 & \langle 1, \cdot\rangle_{L^{2}} \\
0 & \langle\mu(x), \cdot\rangle_{L^{2}} \\
0 & \langle\nu(x), \cdot\rangle_{L^{2}}
\end{array}\right) .
\end{aligned}
$$

To establish the exponential stabilizability of the pair $\left(A_{0}, B_{0}^{*}\right)$, we will refer to [24]. This reference, specifically dealing with the control theory of hyperbolic PDEs, is concerned with the case of control parameters which are function 
of time only, and proves null-controllability results through resolution of moments problems in $L^{2}(0, T)$. Relying on Russel's result of exact controllability in time $T>2 \ell$, we only have to check the following assumptions made on the control that has to take the shape $v(x) u(t)$ :

$\liminf _{n \rightarrow \infty} n\left|\left\langle v, \varphi_{n}\right\rangle_{L^{2}(0, \ell)}\right|>0$ and $\left\langle v, \varphi_{n}\right\rangle_{L^{2}(0, \ell)} \neq 0, \forall n \in \mathbb{N}^{*}$. Since we have indeed control terms that writes $W_{\text {mod }}$, $\mu(x) u_{b}(t)$ and $\nu(x) w_{b}^{\prime \prime}(t)$, and since even with the simple control term $\nu w_{b}^{\prime \prime}$ we obtain:

$$
\liminf _{n \rightarrow \infty} n\left|\left\langle\nu, \varphi_{n}\right\rangle_{L^{2}}\right|=\frac{\sqrt{2 \ell}}{\pi} \text { and }\left\langle\nu, \varphi_{n}\right\rangle_{L^{2}} \neq 0, \forall n \in \mathbb{N}^{*},
$$

then the assumption on the pair $\left(A_{0}, B_{0}\right)$, thus on the pair $\left(A, B_{1}\right)$ is proved, thanks to this $w_{b}^{\prime \prime}$ control contribution.

Remark 3: To re-emphasise Remark 2, since the calculation of $\left\langle\mu, \varphi_{n}\right\rangle_{L^{2}}$ gives $2 \sqrt{2 \ell}\left(1-(-1)^{n}\right) / n^{3} \pi^{3}$, we can prove that the $u_{b}$ control has no influence on even-indexed modes.

\section{TOWARDS NUMERICAL Simulations}

As previously demonstrated, a state-space based controller for the infinite-dimensional $\mathcal{H}_{\infty}$-control problem may be calculated by solving two Riccati equations. However, these equations can rarely be solved exactly [13], [26]. Therefore, we choose to approximate the original infinite-dimensional system by a sequence of finite-dimensional systems that can be robustly controlled with the usual tools of automatic control: a modal decomposition of our linear PDE model is performed, so that system (9) becomes a classical state space system suitable for simulations. The truncation of the PDE system proposed hereafter can be seen as a way of coming back to the structural vibrations of the system. In particular, since we have the robust control result in infinite dimension, we should be able to consider as many modes as needed. Note that this early lumping approach can not be corroborated by the convergence result [13] since the observation operator is unbounded.

\section{A. Finite dimensional model, by modal truncation}

Let us consider the Hermitian base $\left(\varphi_{n}\right)_{n \in \mathbb{N}^{*}}$ of $L^{2}(0, \ell)$ defined in (12) and given by the eigenfunctions of the compact self-adjoint operator $\frac{T_{s}}{\rho \mathcal{A}} \partial_{x x}$. For all $x \in(0, \ell)$ and $n \in \mathbb{N}^{*}$, we have: $\frac{T_{s}}{\rho \mathcal{A}} \partial_{x x} \varphi_{n}(x)=-\omega_{n}^{2} \varphi_{n}(x)$, where $\omega_{n}=\frac{n \pi}{\ell} \sqrt{\frac{T_{s}}{\rho \mathcal{A}}}$. The modal decomposition is achieved through the separation of variables, which meets the Galerkin method [15, chap 7]. The modal in-plane movement $w_{m}$ can be decomposed as follows:

$w_{m}(x, t)=\sum_{n=1}^{+\infty} z_{n}(t) \varphi_{n}(x)$, where $z_{n}(t)=\left\langle w_{m}(\cdot, t), \varphi_{n}\right\rangle_{L^{2}}$.

Since initial conditions are assumed equal to zero, we have $z_{n}(0)=z_{n}^{\prime}(0)=0, \forall n \geq 1$.

The first step is to rewrite the modal equation (7) as a linear system of ordinary differential equations in $\left(z_{n}\right)_{n \geq 1}$. Note that the viscous damping term will be translated in a modal damping shape in the process: $\xi \partial_{t} w_{m}=\sum_{n=1}^{\infty} 2 \omega_{n} \xi_{n} z_{n}^{\prime}(t) \varphi_{n}(x)$ where $\xi_{n}<1$ is the ratio of the actual damping over the critical damping. In an unperturbed hyperbolic system, the critical damping represents the smallest amount of damping for which no oscillation occurs in the free vibration response. Therefore, by projection on the chosen Hermitian base:

$$
\begin{array}{r}
\forall n \geq 1, z_{n}^{\prime \prime}(t)=-\omega_{n}^{2} z_{n}(t)-2 \omega_{n} \xi_{n} z_{n}^{\prime}(t) \\
-\frac{\varrho^{2} \mathcal{A}^{2} E}{2 \rho T_{s}^{2}} \sum_{k=1}^{+\infty}\left\langle(\ell-2 x) \partial_{x} \varphi_{k}, \varphi_{n}\right\rangle_{L^{2}} z_{k}(t) \\
+\alpha_{n} u_{b}^{\prime \prime}+\beta_{n} w_{b}^{\prime \prime}+\left(\left(1-\frac{\alpha^{2}}{2}\right) \alpha_{n}+\alpha \beta_{n}\right) \mathbf{u}^{\prime \prime},
\end{array}
$$

where $\alpha_{n}=\frac{\varrho E_{q} \ell \mathcal{A}^{2}}{2 T_{s}^{2}}\left\langle\frac{x}{\ell}-\left(\frac{x}{\ell}\right)^{2}, \varphi_{n}\right\rangle_{L^{2}}, \beta_{n}=\left\langle-\frac{x}{\ell}, \varphi_{n}\right\rangle_{L^{2}}$.

The measurement output $Y$ then becomes:

$$
Y(t)=\frac{\mathcal{A} E_{q}}{\ell} u_{b}(t)-\frac{\varrho \mathcal{A}^{2} E \ell}{2 T_{s}} \sum_{n=1}^{+\infty} z_{n}(t) \partial_{x} \varphi_{n}(\ell) .
$$

Given $N \in \mathbb{N}^{\star}$, we can thus build a finite dimensional model using the truncated basis $\left(\varphi_{n}\right)_{1 \leq n \leq N}$ of the $N$ first modes. As in Section II-C, the control input is the acceleration of the displacement actuator: $U=\mathbf{u}^{\prime \prime} \in \mathbb{R}$. The choice of the state variables is not unique but numerically it is convenient to choose: $X_{N}=\left(z_{1}^{\prime}, \omega_{1} z_{1}, \ldots, z_{N}^{\prime}, \omega_{N} z_{N}\right) \in \mathbb{R}^{2 N}$.

Be aware of the difference with $X=\left(w_{m}, \partial_{t} w_{m}\right)$. The finite dimensional model takes the usual shape:

$$
\left\{\begin{array}{l}
X_{N}^{\prime}=A_{N} X_{N}+B_{1, N} W+B_{2, N} U \\
Z_{N}=C_{1, N} X_{N}+D_{12, N} U \\
Y_{N}=C_{2, N} X_{N}+D_{21, N} W
\end{array}\right.
$$

with $X_{N}(0)=0$, and where the operators of system $(9)$ are replaced by real-valued matrices $A_{N}, \ldots D_{12, N}$ computed on a truncated basis $\left(\varphi_{n}\right)_{n=1, \ldots, N}$. The measurement output $Y_{N}$ is obtained by truncation of $Y$ on the first $N$ vibration modes. The controlled output vector $Z_{N}$ will be defined accordingly to the expected performance objectives. The exogenous perturbation vector $W=\left(W_{\text {mod }}, u_{b}, w_{b}^{\prime \prime}\right) \in \mathbb{R}^{3}$ remains unchanged.

The advantage of this representation is that all the variables in $X_{N}$ express a velocity, and in (15), $A_{N}$ is dimensionally homogeneous, improving the conditioning of the system.

Let us now define precisely the matrices involved in (15). The dynamic matrix $A_{N}$, of size $2 N \times 2 N$, is given by: $A_{N}=\operatorname{block}_{n, k}\left(\left[\begin{array}{cc}-2 \omega_{n} \xi_{n} \delta_{n k} & a_{n k} \\ \omega_{n} \delta_{n k} & 0\end{array}\right]\right)$, where $\delta_{n k}$ is the Kronecker symbol, $\xi_{n}$ are the modal damping ratios and $a_{n k}=$ $-\omega_{n} \delta_{n k}-\frac{\varrho^{2} \mathcal{A}^{2} E}{2 \omega_{k} \rho T_{s}^{2}}\left\langle(\ell-2 x) \partial_{x} \varphi_{k}, \varphi_{n}\right\rangle$. The choice of diagonal matrices corresponds to a decoupling assumption of the different modes. They could refer for instance to the neglected non-linearities. We also define: $B_{1, N}=\operatorname{vect}_{n}\left[\begin{array}{ccc}d_{n}^{1}-\omega_{u}^{2} \alpha_{n} & \beta_{n} \\ 0 & 0 & 0\end{array}\right]$, $B_{2, N}=\operatorname{vect}_{n}\left[\left(1-\frac{\alpha^{2}}{2}\right) \alpha_{n}+\alpha \beta_{n}\right], C_{2, N}=\left(\operatorname{vect}_{k}\left[\begin{array}{c}c_{k} \\ 0\end{array}\right]\right)^{\top}$ and $D_{21, N}=\left[d_{2} \frac{\mathcal{A} E_{q}}{l} 0\right]$, where $c_{k}=-\frac{\varrho \mathcal{A}^{2} E \ell}{2 \omega_{k} T_{s}} \partial_{x} \varphi_{k}(\ell)$. The parameters $d_{n}^{1}, d^{2} \in \mathbb{R}$ are tuning parameters defining the respective weights of the disturbance signals. Finally, depending on the control objectives of performance, we can choose for instance to stabilize each of the $N$ first modes of vibration and the amplitude of the control, i.e.: $Z_{N}=\left(z_{1}, \ldots, z_{N}, \mathbf{u}^{\prime \prime}\right)$. 
However, in practice we want to control not only the vibrations of the cable, but also reasonably limit the actuator displacement. For this purpose, the to-be-controlled output will be chosen as $Z_{N}=\left(z_{1}, \ldots, z_{N}, \mathbf{u}, \mathbf{u}^{\prime \prime}\right)$ and the control $U$ is of the form $U(t)=\mathbf{u}^{\prime \prime}(t)=-k_{p} \mathbf{u}^{\prime}(t)-k_{i} \mathbf{u}(t)+V(t)$, where $k_{p}, k_{i}>0$ are feedback gains constants and $V(t)$ denotes the strain exerted on the cable by the active tendon. This is a Proportional and Integral + a strain feedback control law. To deal with the chosen control structure, we introduce the augmented state variable: $\widetilde{X}_{N}=\left(X_{N}, \mathbf{u}, \mathbf{u}^{\prime}\right)$. The finite dimensional model reads:

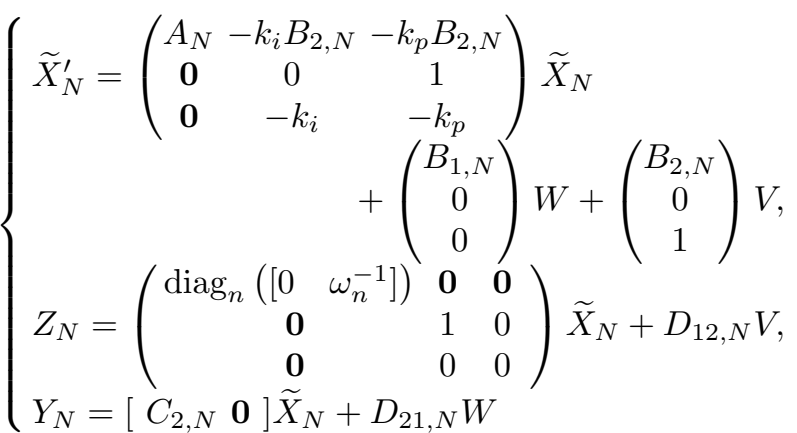

where $D_{12, N}=\left(\begin{array}{lll}\mathbf{0} & 0 & 1\end{array}\right)^{\top}$ and we seek an output feedback controller $\left(k_{p}, k_{i}, K\right)$ where the controller state $X_{K} \in \mathbb{R}^{n_{K}}$ follows $\left[\begin{array}{c}\dot{X}_{K} \\ V\end{array}\right]=\left[\begin{array}{cc}A_{K} & B_{K} \\ C_{K} & D_{K}\end{array}\right]\left[\begin{array}{c}X_{K} \\ Y\end{array}\right]$ so that the closed-loop system satisfies the two following properties: internal stability and optimal $H_{\infty}$ performance. In what follows we choose to synthesize full-order controllers, i.e. of the same order of the to-be-controlled system. Here, the order of the controller is $n_{K}=2 N+2$ but this choice is not limiting, as reduced order controllers could be synthetized.

\section{B. Mixed PI/strain-control simulations}

Following [4], we simulate a $\ell=1.98 \mathrm{~m}$ long steel cable inclined at $\theta=20$ degrees to the horizontal. It has a diameter of $0.8 \mathrm{~mm}$ and a mass of $0.67 \mathrm{~kg} \cdot \mathrm{m}^{-1}$. We have: $\rho=1.34 \times$ $10^{6} \mathrm{~kg} . \mathrm{m}^{-3}, \mathcal{A}=0.5 \times 10^{-6} \mathrm{~m}^{3}, T_{s}=205 \mathrm{~N}$ and $E=200 \times$ $10^{9} \mathrm{~N} . \mathrm{m}^{-2}$. This yields the parameters $E_{q}=174 \times 10^{9} \mathrm{~N} . \mathrm{m}^{-2}$, $\lambda^{2}=1.74$ and $\varrho=\rho g \cos \theta=12.35 \times 10^{6} \mathrm{~kg} \cdot \mathrm{s}^{-2} \cdot \mathrm{m}^{-2}$. These values match at best a typical full-scale bridge cable of length $400 \mathrm{~m}$, mass per unit length $130 \mathrm{~kg} \cdot \mathrm{m}^{-1}$ and tension $8000 \mathrm{kN}$. We take $\alpha=0.1 \mathrm{rad}$, reasonable estimation of the tendon's angle. The first theoretical natural frequencies of inplane vibration modes of the cable are: $\omega_{1}=27.7, \omega_{2}=55.5$, $\omega_{3}=83.3 \mathrm{rad} . \mathrm{s}^{-1}$ and a realistic mean value of the cable's damping ratio is taken as $\xi_{n}=0.2 \%$. We choose $\omega_{u} \simeq \omega_{1}$ to ensure disturbance rejection near the vibration mode we want to dampen the most. Besides, the respective weights of the disturbance signals are chosen as: $d_{n}^{1}=10^{-3}$ and $d^{2}=$ $10^{-3}$. The cable is excited vertically at its bottom support. Two different excitations are considered hereafter: a step excitation or a sinusoidal excitation $u_{b}(t)=\cos (\Omega t) \sin (\theta), w_{b}(t)=$ $\cos (\Omega t) \cos (\theta)$. No external forces are applied on the cable.

All our computations are done with hinfstruct from the MATLABC Robust Control Toolbox, which specifically enables us to deal with the best tuning of parameters $k_{i}, k_{p}$.

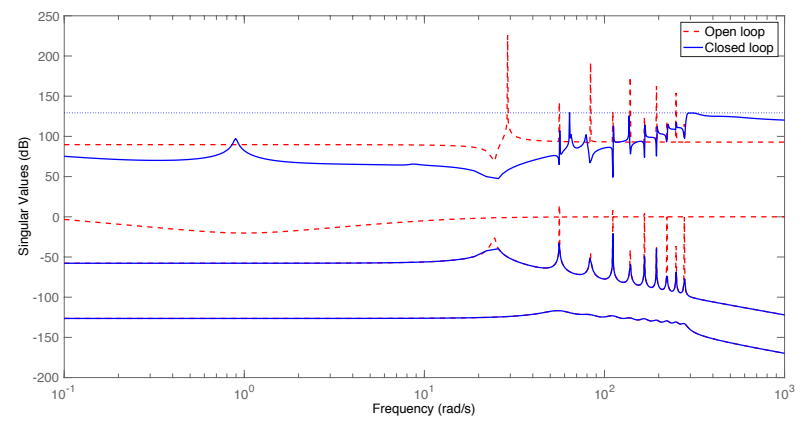

Fig. 2. Open and closed loop time response of the ten first modes to a step excitation with no damping.

We observe in Figure 2 that the first and most important mode is well attenuated. The effect in closed-loop of the synthesized controller is shown in time domain (and compared to the open-loop) considering the step excitation (Figure 3) or a sinusoidal excitation (Figure 4): the vibration reduction is clearly visible from the beginning of the control action.
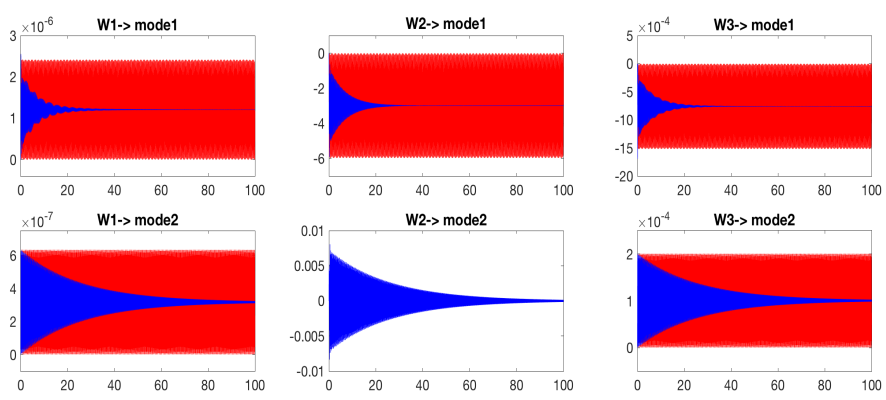

Fig. 3. Open and closed loop time response of the two first modes to a step excitation on each perturbation input $W_{i}, i=1,2,3$, with no damping $\left(\xi_{n}=0\right) . k_{p}=7.91, k_{i}=3.84$.
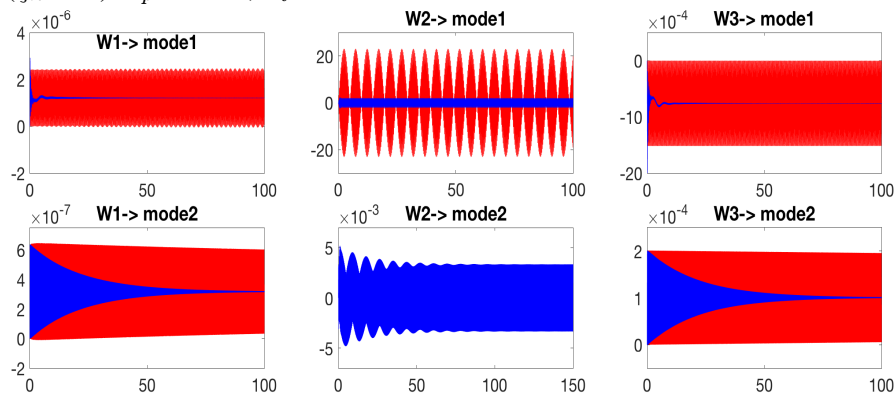

Fig. 4. Open and closed loop time response of the two first modes applying a step excitation on the first input $W_{1}$, and sinusoidal excitations on the second and third inputs, $W_{2}$ and $W_{3}$, with no damping. $k_{p}=0.99, k_{i}=1.27$.

Figure 3 specifically shows that the damping time scale of each mode is quite different between even and odd modes. Following Remark 2, this corroborates the comparison between the effect of inertial and parametric control in [7]. As expected due to the definition of $(15)$ and Remark 3, the perturbation $W_{2}=u_{b}$ has no influence on even-indexed modes: the closed loop result shows only the control action. We observe that, as expected, the amplitude of the actuator displacement remains bounded within physically reasonable limits ; see Figure 5. 

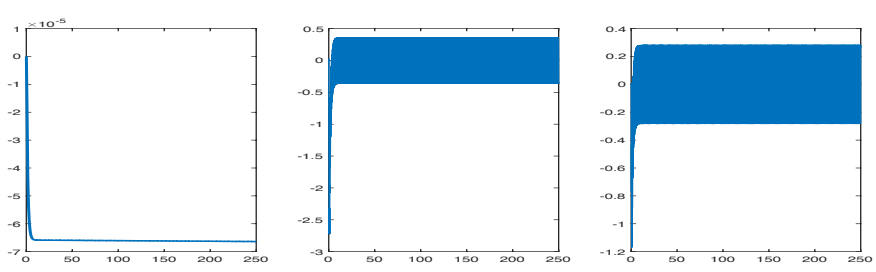

Fig. 5. Closed-loop time response of the actuator displacement, for $N=2$, applying a step excitation on the first input $W_{1}$, and sinusoidal excitations on the second and third inputs, $W_{2}$ and $W_{3}$ with no damping.

We conclude this section with some observations about the spillover effect by implementing the $H_{\infty}$ optimal full order controller $K$ synthetized for $N$ modes, into a plant of larger order. It is well-known that for vibration systems (covered by wave or plate PDEs), at least the first neglected mode is actually excited by the controller of all the previous ones [9], [26]. Here, we numerically observe that the control synthesized for $N=3$ modes fails to stabilize the 4 th mode as shown on Figure 6 In practice, this effect is easily avoided as soon as a small damping is included in the system. By trial and error, we observe that a damping ratio ten times less than the realistic one, is enough to prevent the spillover effect.
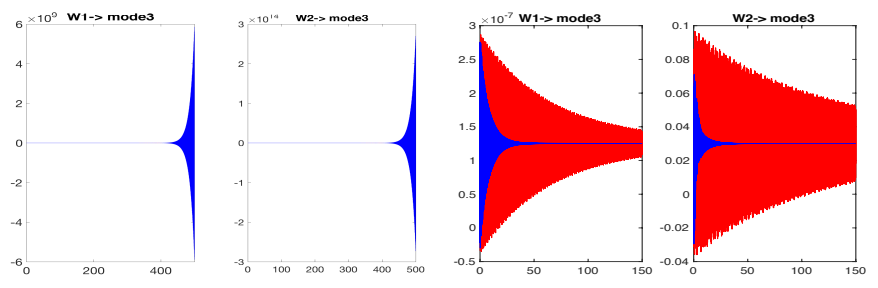

Fig. 6. Closed-loop time response of the $3 t h$ mode applying a step excitation on the first input $W_{1}$, and a sinusoidal excitation on the second input $W_{2}$ when using the robust controller synthesized for $N=2$ modes. Left: spillover with the lack of damping. Right: no spillover with a small damping $\left(\xi_{n}=0.2 \%\right)$.

Lastly, note that another strength of the present approach is to deal with as many modes as needed. In practice, civil engineers typically deal with two or three modes (often to be able to keep track of the nonlinear couplings, which are not considered here). As illustrated on Figure 2, we can, for example, robustly control the ten first modes of the cable.

\section{Conclusion}

In this article, based on a PDE modeling of a cable, we were able to perform an infinite dimensional robust control analysis of the vibration reduction of a highly flexible system. Taking advantage of our specific approach and based on the truncation of our PDE model, the numerical simulations allow to deal either with the first few modes (for instance in order, later, to be able to being compared with results from e.g. [4], [1], [3]), or with a lot of modes, which is not usually possible when considering non-linearities for instance. In both cases, the numerical illustrations shows the efficiency of the robust control performed on the system, from localized measurements and control actions.

\section{REFERENCES}

[1] M. Smrz, R. Bastaits, and A. Preumont, "Active damping of the camera support mast of a cherenkov gamma-ray telescope," Nuclear Instruments and Meth. in Physics Research Sec. A, vol. 635, no. 1, pp. 44-52, 2011.

[2] H. Irvine, "Cable structures", MIT Press series in structural mechanics, 1981.

[3] P. Warnitchai, Y. Fujino, and T. Susumpow, "A non-linear dynamic model for cables and its application to a cable structure system," Journal of Sound and Vibration, vol. 187, no. 4, pp. 695 - 712, 1995.

[4] A. Gonzalez-Buelga, S. Neild, D. Wagg, and J. Macdonald, "Modal stability of inclined cables subjected to vertical support excitation," Journal of Sound and Vibration, vol. 318, no. 3, pp. 565 - 579, 2008.

[5] J. Macdonald, M. Dietz, S. Neild, A. Gonzalez-Buelga, A. Crewe, and D. Wagg, "Generalised modal stability of inclined cables subjected to support excitations." Journal of Sound and Vibration, vol. 329, no. 21, pp. 4515-4533, 2010.

[6] G. Song, V. Sethi, and H.-N. Li, "Vibration control of civil structures using piezoceramic smart materials: A review," Engineering Structures, vol. 28 , no. 11 , pp. 1513 - 1524, 2006.

[7] A. Preumont, Vibration control of active structures: an introduction, ser. Solid Mechanics and its Applications, 1997, vol. 50.

[8] Y. Fujino and T. Susumpow, "An experimental study on active control of in-plane cable vibration by axial support motion," Earthquake Engineering and Structural Dynamics, vol. 23, no. 12, pp. 1283-1297, 1994.

[9] G. J. Balas and J. C. Doyle, "Robustness and performance tradeoffs in control design for flexible structures," in Proceedings of the 29th IEEE Conference on Decision and Control. IEEE, 1990, pp. 2999-3010.

[10] L. Baudouin, S. Neild, A. Rondepierre, and D. Wagg, "Robust measurement feedback control of an inclined cable," in Proceedings of the 1st IFAC Workshop on Control of Systems Modeled by PDEs, 2013.

[11] B. van Keulen, " $H_{\infty}$-control with measurement-feedback for PritchardSalamon systems," Internat. J. Robust Nonlinear Control, vol. 4, no. 4, pp. 521-552, 1994.

[12] A. Bensoussan and P. Bernhard, "On the standard problem of $H_{\infty}$ optimal control for infinite-dimensional systems," in Identification and control in systems governed by pdes. SIAM, 1993, pp. 117-140.

[13] K. Morris, " $H_{\infty}$-output feedback of infinite-dimensional systems via approximation," Sys. \& Control Let., vol. 44, no. 3, pp. 211-217, 2001.

[14] F. Bossens and A. Preumont, "Active tendon control of cable-stayed bridges: a large-scale demonstration," Earthquake Engineering \& Structural Dynamics, vol. 30, no. 7, pp. 961-979, 2001.

[15] D. Wagg and S. Neild, Nonlinear Vibration with Control: For Flexible and Adaptive Structures, ser. Solid mechanics and appli. Springer, 2010.

[16] R. F. Curtain and H. Zwart, An introduction to infinite-dimensional linear systems theory, ser. Texts in Applied Mathematics. Springer-Verlag, 1995, vol. 21

[17] M. Krstic, B.-Z. Guo, A. Balogh, and A. Smyshlyaev, Output-feedback stabilization of an unstable wave equation, Automatica, vol. 44, pp. 63-74, 2008.

[18] A. Preumont and F. Bossens, "Active tendon control of cable-stayed bridges," SPIE proceedings series, vol. Smart Structures and Materials, pp. 188-198, 2000.

[19] J. C. Doyle, K. Zhou, and K. Glover, Robust and Optimal Control. Prentice Hall, 1996.

[20] M. Tucsnak and G. Weiss, Observation and Control for Operator Semigroups, ser. Birkäuser Advanced Texts. Springer, 2009, vol. XI.

[21] B. van Keulen, " $H_{\infty}$-control with measurement-feedback for linear infinite-dimensional systems," J. Math. Systems Estim. Control, vol. 3, no. 4, pp. 373-411, 1993.

[22] K. Mikkola and O. Staffans, "A Riccati equation approach to the standard infinite-dimensional $H_{\infty}$ problem," in Proceedings of the Mathematical Theory of Networks and Systems (MTNS), 2002.

[23] V. Barbu, " $H_{\infty}$ boundary control with state feedback: the hyperbolic case,” SIAM J. Control Optim., vol. 33, no. 3, pp. 684-701, 1995.

[24] D. L. Russell, "Nonharmonic Fourier series in the control theory of distributed parameter systems," J. Math. Anal. Appl., vol. 18, pp. 542560, 1967.

[25] V. Komornik, "A new method of exact controllability in short time and applications," Ann. Fac. Sci. Toulouse Math. (5), vol. 10, no. 3, pp. 415-464, 1989.

[26] K. Morris, "Control of Systems Governed by Partial Differential Equations," in The Control Handbook, 2nd ed., ser. Electrical Engineering Handbook, W. S. Levine, Ed. CRC press, 2010, vol. 3, ch. XI. 